\title{
A FOCK SPACE MODEL FOR ADDITION AND MULTIPLICATION OF C-FREE RANDOM VARIABLES
}

\author{
MIHAI POPA \\ (Communicated by Marius Junge)
}

ABstract. The paper presents a Fock space model suitable for construction of c-free algebras. Immediate applications are direct proofs for the properties of the c-free $R$ - and $S$-transforms.

\section{INTRODUCTION}

Two important tools in free probability theory are the $R$ - and $S$-transforms which play a similar role to the Fourier, respectively Mellin, transform. More precisely, besides strong regularity properties, if $X$ and $Y$ are two free non-commutative random variables, then $R_{X+Y}(z)=R_{X}(z)+R_{Y}(z)$ and $S_{X Y}(z)=S_{X}(z) \cdot S_{Y}(z)$ if $X, Y$ have non-zero first order moments.

In literature there are two main techniques to prove the additive, respectively multiplicative, properties of the $R$ - and $S$-transforms. The proofs given by D.V. Voiculescu ([19], 20]) and U. Haagerup ([10]) are based on functional analysis techniques, namely on the properties of the annihilation and creation operators on the full Fock space, while the proofs of R. Speicher and A. Nica ([13]) are based on combinatorial techniques on the lattice of non-crossing partitions (also, noncrossing linked partitions appear in the proofs for the multiplicative property of the $S$-transform in [8, 16]).

In the early 1990's, M. Bozejko, M. Leinert and R. Speicher introduced the notion of $c$-freeness, which extends the notion of freeness to the framework of and algebra endowed with two $(\phi, \psi)$, rather than one, normalized linear functionals (see Section 2 for the exact definitions). (A more general approach to c-freeness, considering pairs of completely positive maps and conditional expectations, has been pursued by F. Boca ([3]), K. Dykema and E. Blanchard ([9]), M. Popa, V. Vinnikov ([14, 17], etc.). The addition of c-free random variables was studied in 7 (and later in [1], 22], 11], etc.), where a c-free version of the $R$-transform, the ${ }^{c} R$-transform, with similar additivity and analytic properties (for $\phi=\psi$, the two transforms coincide) was constructed. A multiplication of c-free random variables was studied in [18, where a c-free extension of the $S$-transform was constructed. In both cases, the proofs of the key properties (addition for the ${ }^{c} R$ - and multiplication for the ${ }^{c} S$-transform) are combinatorial, much like the proofs from [13], heavily relying on the properties on non-crossing partitions. The present material gives

Received by the editors February 17, 2011 and, in revised form, March 24, 2012.

2010 Mathematics Subject Classification. Primary 46L54, 30H20.

Key words and phrases. Free independence, c-free independence, creation/annihilation operators, ${ }^{c} R$ - and ${ }^{c} S$-transforms. 
a new approach to c-free random variables, in the spirit of the construction from [10]. Particularly, we give a more direct proof of the additive and multiplicative properties of the ${ }^{c} R$ - and ${ }^{c} S$-transforms, based on the properties of the creation and annihilation operators on a certain type of Fock space.

Besides the Introduction, the paper is organized in 3 sections. Section 2 presents basic definitions, the construction of the space $\mathcal{E}(\mathfrak{H}, \mathcal{T}(\mathcal{K}))$ and an operator algebra model for c-free algebras. Section 3 presents the construction of some operators of prescribed ${ }^{c} R$ and ${ }^{c} S$-transforms, and Section 4 gives the proof for the additive, respectively multiplicative, properties of ${ }^{c} R$ and ${ }^{c} S$.

In this paper, rather than the $S$ - or ${ }^{c} S$-transforms, we use, to simplify the notation, their multiplicative inverses, the so-called $T$-and ${ }^{c} T$-transforms, i.e. $T_{X}(z) \cdot S_{X}(z)=1$ (see [8], [16]), respectively ${ }^{c} T(z) \cdot{ }^{c} S(z)=1$ (see [18]).

\section{A CONStruction of C-FREe Algebras}

2.1. Suppose $\mathcal{A}$ is a complex unital algebra endowed and $\psi: \mathcal{A} \longrightarrow \mathbb{C}$ is a linear map such that $\varphi(1)=1$. A family of unital subalgebras $\left\{\mathcal{A}_{i}\right\}_{i \in I}$ of $\mathcal{A}$ is said to be free (with respect to $\psi$ ) if

$$
\psi\left(x_{1} \cdots x_{n}\right)=0
$$

whenever $x_{j} \in \mathcal{A}_{\epsilon(j)}$ with $\epsilon(k) \neq \epsilon(k+1)$ and $\psi\left(x_{j}\right)=0$ for $1 \leq j \leq n$ and $1 \leq k<n$.

If $\phi: \mathcal{A} \longrightarrow \mathbb{C}$ is another linear map with $\phi(1)=1$, the family $\left\{\mathcal{A}_{i}\right\}_{i \in I}$ of unital subalgebras of $\mathcal{A}$ is said to be $c$-free with respect to $(\phi, \psi)$ if $\left\{A_{i}\right\}_{i \in I}$ are free with respect to $\psi$ and

$$
\phi\left(x_{1} \cdots x_{n}\right)=\phi\left(x_{1}\right) \cdots \phi\left(x_{n}\right)
$$

whenever $x_{j} \in \mathcal{A}_{\epsilon(j)}$ with $\epsilon(k) \neq \epsilon(k+1)$ and $\psi\left(x_{j}\right)=0$ for $1 \leq j \leq n$ and $1 \leq k<n$.

Take $X$ to be an element from $\mathcal{A}$ and let $m_{X}(z)=\sum_{k=1}^{\infty} \psi\left(X^{k}\right)$ denote the moment generating series of $X$ with respct to $\psi$. As formal power series, the transforms $R_{X}(z)$ and, if $\psi(X) \neq 0, T_{X}(z)$ are defined by the equations

$$
\begin{aligned}
m_{X}(z) & =R_{X}\left(z\left[1+m_{X}(z)\right]\right), \\
\frac{1}{z} m_{X}(z) & =\left[T_{X}\left(m_{X}(z)\right)\right] \cdot\left(1+m_{X}(z)\right) .
\end{aligned}
$$

We warn the reader that the version of the $R$-transform that is used in the present material differs from the original definition of D.-V. Voiculescu (that we will here call $\mathcal{R}$ ) by a multiplication with the variable $z: R_{X}(z)=z \cdot \mathcal{R}_{X}(z)$. As also seen in [18, 13, this shift of coefficients simplifies the notation in several recurrence relations from subsection 2.2 .

For $\mathcal{H}$ a complex Hilbert space, we define $\mathcal{T}^{0}(\mathcal{H})=\mathcal{H} \oplus(\mathcal{H} \otimes \mathcal{H}) \oplus(\mathcal{H} \otimes \mathcal{H} \otimes \mathcal{H}) \oplus \ldots$ and $\mathcal{T}(\mathcal{H})=\mathbb{C} \omega \oplus \mathcal{T}^{0}(\mathcal{H})$, where $\|\omega\|=1$. For $e \in \mathcal{H}$ a non-zero vector, the creation operator over $e, a_{e}^{*} \in \mathcal{L}(\mathcal{T}(\mathcal{H}))$, is given by the relations

$$
\begin{aligned}
& a_{e}^{*} \omega=e, \\
& a_{e}^{*} v_{1} \otimes v_{2} \otimes \cdots \otimes v_{k}=e \otimes v_{1} \otimes v_{2} \otimes \cdots \otimes v_{k}, \text { for } v_{1}, \ldots, v_{k} \in \mathcal{H},
\end{aligned}
$$

while the annihilation operator over $e, a_{e} \in \mathcal{L}(\mathcal{T}(\mathcal{H}))$, is given by

$$
\begin{aligned}
& a_{e} \omega=0, \\
& a_{e} v_{1} \otimes v_{2} \otimes \cdots \otimes v_{k}=\left\langle v_{1}, \xi\right\rangle \cdot v_{2} \otimes \cdots \otimes v_{k} .
\end{aligned}
$$


We recall the following result (see [21] for (1), 10], Theorem 2.2 and Theorem 2.3, for $(2)$ and $(3))$ :

Theorem 2.1. Let $\mathcal{H}$ be a complex Hilbert space, $e_{1}$ and $e_{2}$ be two orthogonal vectors from $\mathcal{H}$, and $f_{1}, f_{2}$ be polynomials with complex coefficients. For e $\in \mathcal{H} \backslash$ $\{0\}$ we will denote by $\mathcal{A}(e)$ the algebra generated by the creation and annihilation operators over $e$.

(1) The algebras $\mathcal{A}\left(e_{1}\right)$ and $\mathcal{A}\left(e_{2}\right)$ are free with respect to the vacuum state $T \mapsto\langle T \omega, \omega\rangle$.

(2) If $\alpha_{i}=a_{e_{i}}^{*}+f\left(a_{e_{i}}\right)(i=1,2)$, then $R_{\alpha_{i}}(z)=z \cdot f_{i}(z)$ and $R_{\alpha_{1}+\alpha_{2}}(z)=$ $z \cdot f_{1}(z)+z \cdot f_{2}(z)$.

(3) If $f_{i}(0) \neq 0$ and $\beta_{i}=\left[\boldsymbol{I d}_{\mathcal{T}(\mathcal{H})}+a_{e_{i}}^{*}\right] f\left(a_{e_{i}}\right)(i=1,2)$, then $T_{\beta_{i}}(z)=f_{i}(z)$ and $T_{\beta_{1} \beta_{2}}(z)=f_{1}(z) \cdot f_{2}(z)$.

2.2. Now consider two complex Hilbert spaces $\mathcal{K}$ and $\mathfrak{H}$ and $\omega$ a distinguished unit vector in $\mathfrak{H}$. Take $\mathcal{T}(\mathcal{K})=\omega_{1} \oplus \mathcal{T}^{0}(\mathcal{K})$, where again $\left\|\omega_{1}\right\|=1$ and

$$
\mathcal{E}(\mathfrak{H}, \mathcal{K})=\mathfrak{H} \oplus(\mathfrak{H} \otimes \mathcal{T}(\mathcal{K})) .
$$

Later in this section and in Section 4 , we will consider $\mathcal{E}(\mathfrak{H}, \mathcal{K})$ for a particular $\mathfrak{H}$. When there is no possibility of confusion, to simplify the writing, we will use $\mathcal{E}$ for $\mathcal{E}(\mathfrak{H}, \mathcal{K})$. Put $\mathfrak{H}^{0}=\mathfrak{H} \ominus \mathbb{C} \omega, \Omega=\omega \otimes \omega_{1}, \mathcal{E}^{0}=\mathcal{E} \ominus \mathbb{C} \Omega$. We define the following embedding $\pi: \mathcal{L}(\mathcal{K}) \longrightarrow \mathcal{L}(\mathcal{T}(\mathcal{H}))$ :

$$
\pi(a)=a \oplus a \otimes\left(\mathbf{I d}_{\mathfrak{H} \otimes \mathcal{T}^{0}(\mathcal{K})} \oplus 0_{\mathbb{C} \Omega}\right) .
$$

Note that $\pi(\mathcal{L}(\mathfrak{H}))$ has unit $\pi\left(\mathbf{I d}_{\mathfrak{H}}\right)=\mathbf{I d}_{\mathcal{E}_{0}} \neq \mathbf{I d}_{\mathcal{E}}$.

For a non-zero vector $\eta \in \mathcal{K}$ we define the operators $A_{\eta}^{*}$ and $\left\{A_{\eta, n}\right\}_{n \geq 0}$ from $\mathcal{L}(\mathcal{E})$ as follows:

$$
\begin{aligned}
A_{\eta}^{*} \zeta & =0, \text { if } \zeta \in \mathfrak{H} \oplus \mathfrak{H}^{0} \otimes \mathcal{T}(\mathcal{K}), \\
A_{\eta}^{*} \omega \otimes \omega_{1} & =\omega \otimes \eta, \\
A_{\eta}^{*} \omega \otimes \zeta & =\omega \otimes(\eta \otimes \zeta) \text { for all } \zeta \in \mathcal{T}^{0}(\mathcal{K}) .
\end{aligned}
$$

We put $A_{\eta, 0}=\mathbf{I d}_{\mathbb{C} \Omega}$ and, for $n \leq 1$, we define $A_{\eta, n}$ via

$$
\begin{aligned}
& A_{\eta, n} \omega \otimes\left(\eta^{\otimes n}\right)=\omega \otimes \omega_{1}, \\
& A_{\eta, n} \zeta=0, \text { if } \zeta \notin \mathbb{C} \omega_{1} \otimes\left(\eta^{\otimes n}\right), \text { where } \eta^{\otimes n}=\underbrace{\eta \otimes \cdots \otimes \eta}_{n \text { times }} .
\end{aligned}
$$

We will use the notation $\mathcal{D}(\eta)$ for the algebra generated by $A_{\eta}^{*}$ and $\left\{A_{\eta, n}\right\}_{n \geq 0}$. If $\mathcal{A}_{1}$ and $\mathcal{A}_{2}$ are two subalgebras of $\mathcal{L}(\mathcal{E})$, then the notation $\mathcal{A}_{1} \vee \mathcal{A}_{2}$ will stand for the algebra generated by them in $\mathcal{L}(\mathcal{E})$.

Remark 2.2. Fix $\eta, \eta_{0} \in \mathcal{K}$ unit vectors. From the definitions of $\pi, A_{\eta}^{*}, A_{\eta, n}$, trivial verifications give that

$$
A_{\eta, n}\left(A_{\eta}^{*}\right)^{p}= \begin{cases}A_{\eta, n-p} & \text { if } n \geq p, \\ 0 & \text { if } n<p .\end{cases}
$$

If $x \in \pi(\mathcal{L}(\mathfrak{H}))$ and $n \geq 0$, then $x A_{\eta, n}=0$. Also, if $m, n>0$, then

$$
\begin{aligned}
& A_{\eta, n} A_{\eta_{0}, m}=0, \\
& \mathbf{I d}_{\mathbb{C} \Omega} A_{\eta, n}=A_{\eta, n} ; \quad A_{\eta, n} \mathbf{I} \mathbf{d}_{\mathbb{C} \Omega}=0, \\
& A_{\eta, n} \mathbf{I d}_{\mathcal{E}_{0}}=0 ; \quad \mathbf{I d}_{\mathcal{E}_{0}} A_{\eta}^{*}=A_{\eta}^{*} \mathbf{I} \mathbf{d}_{\mathcal{E}_{0}}=A_{\eta}^{*} .
\end{aligned}
$$


Remark 2.3. For $\eta_{1}, \eta_{2} \in \mathcal{K}$, we have that

$$
\text { Range }\left(\left(A_{\eta_{1}}^{*}\right)^{p} A_{\eta_{2}}^{*}\right)=\operatorname{Span}\left\{\omega \otimes \eta_{2} \otimes \eta_{1}^{\otimes p}, \omega \otimes \zeta \otimes \eta_{2} \otimes \eta_{1}^{\otimes p}: \zeta \in \mathcal{T}^{0}(\mathcal{K})\right\} ;
$$

therefore, if $\eta_{1} \perp \eta_{2}$, then

$$
A_{\eta_{1}, n}\left(A_{\eta_{1}}^{*}\right)^{p} A_{\eta_{2}}^{*}=0 .
$$

On $\mathcal{L}(\mathcal{E})$ we consider the functionals $\phi(\cdot)=\langle\cdot \Omega, \Omega\rangle$ and $\psi(\cdot)=\left\langle\cdot \Omega_{1}, \Omega_{1}\right\rangle$.

Lemma 2.4. Suppose $\eta_{1}, \eta_{2} \in \mathcal{K}$ and $x \in \pi(\mathcal{L}(\mathfrak{H}))$. Then

$$
\begin{aligned}
A_{\eta_{1}}^{*} x A_{\eta_{2}}^{*} & =A_{\eta_{1}}^{*} \psi(x) A_{\eta_{2}}^{*}, \\
A_{\eta_{1}, n} x A_{\eta_{2}}^{*} & =A_{\eta_{1}, n} \psi(x) A_{\eta_{2}}^{*} .
\end{aligned}
$$

Proof. Since $A_{\eta_{2}}(\mathcal{E})=\omega_{1} \otimes \mathcal{T}^{0}(\mathcal{K})$, for any $\zeta \in \mathcal{E}$ we have that

$$
\begin{aligned}
& A_{\eta_{2}} \zeta=\omega_{1} \otimes \zeta^{\prime} \text { for some } \zeta^{\prime} \in \mathcal{T}^{0}(\mathcal{K}), \\
& x A_{\eta_{2}} \zeta=\psi(x) \omega_{1} \otimes \zeta^{\prime}+v \otimes \zeta^{\prime} \text { for some } v \in \mathcal{T}^{0}(\mathcal{H}) .
\end{aligned}
$$

But $\mathcal{T}^{0}(\mathcal{H}) \otimes \mathcal{T}^{0}(\mathcal{K}) \subset \operatorname{ker}\left(A_{\eta_{1}}^{*}\right), \operatorname{ker}\left(A_{\eta_{1}, n}\right)$; hence the conclusion.

In the proof of the main result of this section, Theorem 2.6 we will use the following lemma:

Lemma 2.5. Suppose that $\mathcal{A}_{1}$ and $\mathcal{A}_{2}$ are two free independent subalgebras of an algebra $\mathcal{A}$ with respect to some linear map $\varphi$ and that $a_{0}, a_{1}, \ldots, a_{n+1} \in \mathcal{A}_{1}$, $b_{1}, \ldots, b_{n} \in \mathcal{A}_{2}$ are such that $\varphi\left(a_{k}\right)=\varphi\left(b_{k}\right)=0$ for all $1 \leq k \leq n$. Then

$$
\varphi\left(a_{0} b_{1} \cdots b_{n} a_{n+1}\right)=0 .
$$

Proof. Take $d_{j}=a_{j}-\varphi\left(a_{j}\right), j \in\{0, n+1\}$. Then $\varphi\left(d_{j}\right)=0$ and $a_{j}=\varphi\left(a_{j}\right)+d_{j}$; therefore

$$
\begin{aligned}
\varphi\left(a_{0} b_{1} \cdots b_{n} a_{n+1}\right)= & \varphi\left(a_{0}\right) \varphi\left(a_{0} b_{1} \cdots b_{n} d_{n+1}\right)+\varphi\left(a_{0}\right) \varphi\left(a_{0} b_{1} \cdots b_{n}\right) \varphi\left(a_{n+1}\right) \\
& +\varphi\left(d_{0} b_{1} \cdots b_{n} d_{n+1}\right)+\varphi\left(d_{0} b_{1} \cdots b_{n}\right) \varphi\left(a_{n+1}\right),
\end{aligned}
$$

and all the above four terms cancel from the definition of free independence.

Theorem 2.6. Let $\mathcal{A}_{1}, \mathcal{A}_{2}$ be two subalgebras of $\mathcal{L}(\mathfrak{H})$ which are free independent with respect to $\psi$ and let $\eta_{1}, \eta_{2}$ be two orthogonal unit vectors $\mathcal{K}$. Then the algebras $\mathfrak{A}_{1}=\pi\left(\mathcal{A}_{1}\right) \vee \mathcal{D}\left(\eta_{1}\right)$ and $\mathfrak{A}_{2}=\pi\left(\mathcal{A}_{2}\right) \vee \mathcal{D}\left(\eta_{2}\right)$ are $c$-free with respect to $(\phi, \psi)$.

Proof. It suffices to prove that for $x_{1}, \ldots, x_{m}$ such that $x_{j} \in \mathcal{A}\left(e_{\epsilon(j)}, \eta_{\epsilon(j)}\right)$ with $\epsilon(i) \neq \epsilon(i+1)$ and $\psi\left(x_{k}\right)=0$, we have

$$
\begin{aligned}
& \psi\left(x_{m} \cdots x_{2} x_{1}\right)=0, \\
& \phi\left(x_{m} \cdots x_{2} x_{1}\right)=\phi\left(x_{m}\right) \cdots \phi\left(x_{2}\right) \phi\left(x_{1}\right) .
\end{aligned}
$$

Note that $\mathcal{T}(\mathcal{H}) \otimes \mathcal{T}(\mathcal{K}) \perp \Omega_{1}$ and

$$
\begin{aligned}
& \mathcal{D}\left(\eta_{i}\right)(\mathcal{E}) \subseteq \mathcal{T}(\mathcal{H}) \otimes \mathcal{T}(\mathcal{K}), \\
& \pi\left(\mathcal{A}_{i}\right)(\mathcal{T}(\mathcal{H}) \otimes \mathcal{T}(\mathcal{K})) \subseteq \mathcal{T}(\mathcal{H}) \otimes \mathcal{T}(\mathcal{K}) .
\end{aligned}
$$

Hence $\psi$ cancels on all reduced products from $\mathfrak{A}_{1} \cup \mathfrak{A}_{2}$ that contain factors from $\mathcal{D}\left(\eta_{1}\right)$ or $\mathcal{D}\left(\eta_{2}\right)$. It follows that we only need to prove the relation (2) for $x_{1}, \ldots, x_{m}$ $\in \pi\left(\mathcal{A}_{1}\right) \cup \pi\left(\mathcal{A}_{2}\right)$, the statement which is equivalent to the free independence of $\mathcal{A}_{1}$ and $\mathcal{A}_{2}$. 
We will prove (3) by induction on $n$. For $n=1$, the assertion is trivial. For the induction step, it suffices to prove that

$$
\phi\left(x_{n} \cdots x_{1}\right)=\phi\left(x_{n}\right) \phi\left(x_{n-1} \cdots x_{1}\right) .
$$

Taking $x_{n}^{\prime}=x_{n}-\phi\left(x_{n}\right) \mathbf{I} \mathbf{d}_{\mathbb{C} \Omega}$, we have that $\phi\left(x_{n}^{\prime}\right)=0$; hence (4) is equivalent to $\phi\left(x_{n} \cdots x_{1}\right)=0$ whenever $\phi\left(x_{n}\right)=0$.

Suppose $x_{n} \in \mathcal{A}_{1} \vee \mathcal{D}\left(\eta_{1}\right)$. Then $x_{n}$ is a linear combination of monomials in elements from $\mathcal{A}_{1}$ and $\mathcal{D}\left(\eta_{1}\right)$. From Lemma 2.4, we can suppose that all factors from $\mathcal{D}\left(\eta_{1}\right)$ are consecutive, so $x_{n}$ is a sum of elements from $\mathcal{A}_{1}$ and monomials of the types $y_{1}^{\prime}\left(A_{\eta_{1}}^{*}\right)^{p} A_{\eta_{1}, m} y_{1}$ or $y_{1}^{\prime}\left(A_{\eta_{1}}^{*}\right)^{p} y_{1}$, with $y_{1}^{\prime}, y_{1} \in \mathcal{A}_{1} \cup \mathbf{I d}$ and $p \geq 0$. If $y_{1}^{\prime} \neq \mathbf{I d}$ or $p \neq 0$, then $x_{n}(\mathcal{E}) \perp \Omega$; hence $\phi\left(x_{n} \cdots x_{1}\right)=0$. Also, if $m=0$, then either $y_{1}=\mathbf{I d}$ and $\phi\left(x_{n}\right) \neq 0$ or $y_{1} \in \mathcal{A}_{1}$ and $x_{n}=0$. Therefore we can suppose that $x_{n}=A_{\eta_{1}, m} y_{1}$ for some $m>0$ and $y_{1} \in \mathcal{A}_{1}$ and that all other $x_{j}$ are either elements of $\mathcal{A}_{\epsilon(j)}$ or monomials as above.

Let $k=\max \left\{j: x_{j}\right.$ contains $\left.A_{\epsilon(j)}^{*}\right\}$ and $p=\max \left\{j: x_{j}\right.$ contains $\left.A_{\epsilon(j)}\right\}$. If $p>k$, then $x_{n} \cdots x_{p}=A_{\eta_{1}, m} y A_{\eta_{\epsilon(p)}} y^{\prime}$, for some $y \in \mathcal{A}_{1} \vee \mathcal{A}_{2}$ and $y^{\prime} \in \mathcal{A}_{\epsilon(p)} \vee \mathcal{D}\left(\eta_{\epsilon(j)}\right)$. From Lemma 2.4 and Remark 2.2, $A_{\eta_{1}, m} y A_{\eta_{\epsilon(p)}}=A_{\eta_{1}, m} \psi(y) A_{\eta_{\epsilon(p)}}=0$.

Suppose that $p \leq k$. If $\epsilon(k)=2$, then $x_{n} \cdots x_{k}=A_{\eta_{1}, m} y A_{\eta_{2}}^{*} y^{\prime}$, for some $y \in \mathcal{A}_{1} \vee \mathcal{A}_{2}$ and $y^{\prime} \in \mathcal{A}_{2} \vee \mathcal{D}\left(\eta_{2}\right)$. Applying Lemma 2.4 and Remark 2.3, we have

$$
x_{n} \cdots x_{k}=A_{\eta_{1}, m} \psi(y) A_{\eta_{2}}^{*} y^{\prime}=0 .
$$

If $\epsilon(j)=1$, then, from Remark 2.3 ,

$$
\begin{aligned}
x_{n} \cdots x_{k} & =A_{\eta_{1}, m} y_{1} x_{n-1} \cdots x_{k+1} y A_{\eta_{1}}^{*} y^{\prime} \\
& =A_{\eta_{1}, m} \psi\left(y_{1} x_{n-1} \cdots x_{k+1} y\right) A_{\eta_{1}}^{*} y^{\prime},
\end{aligned}
$$

but $\psi\left(y_{1} x_{n-1} \cdots x_{k+1} y\right)=0$ from Lemma 2.5. so the proof is complete.

Corollary 2.7. With the notation from subsection 2.1, take $\mathfrak{H}=\mathbb{C} \omega \oplus \mathcal{T}^{0}(\mathcal{H})$, where $\mathcal{H}$ is a complex Hilbert space of dimension at least 2.

Let $e_{1}, e_{2}$, respectively $\eta_{1}, \eta_{2}$, be two pairs of orthogonal unit vectors from $\mathcal{H}$, respectively $\mathcal{K}$. Then the algebras $\mathcal{D}\left(\eta_{1}\right) \vee \pi\left(\mathcal{A}\left(e_{1}\right)\right)$ and $\mathcal{D}\left(\eta_{2}\right) \vee \pi\left(\mathcal{A}\left(e_{2}\right)\right)$ are c-free with respect to the maps $\phi$ and $\psi$ considered above.

Proof. From Theorem 2.1 $(1)$, the algebras $\mathcal{A}\left(e_{1}\right)$ and $\mathcal{A}\left(e_{2}\right)$ are free in $\mathcal{L}(\mathcal{T}(\mathcal{H}))$ with respect to $\langle\cdot \omega, \omega\rangle$, and the conclusion follows from Theorem 2.6 .

\section{ThE ${ }^{c} R$ - AND ${ }^{c} T$-TRANSFORMS}

Consider an algebra $\mathcal{A}$ with two states $\phi, \psi: \mathcal{A} \longrightarrow \mathbb{C}$ and $X \in \mathcal{A}$. Let $m_{X}(z)=$ $\sum_{k=1}^{\infty} \psi\left(X^{k}\right)$, respectively $M_{X}(z)=\sum_{k=1}^{\infty} \phi\left(X^{k}\right)$, be the moment-generating series of $X$ with respect to $\psi$, respectively $\phi$. We define the ${ }^{c} R$ - and, if $\psi(X) \neq 0$, the ${ }^{c} T$-transforms of $X$ by the following equations:

$$
\begin{aligned}
{ }^{c} R_{X}\left(z\left[1+m_{X}(z)\right]\right) \cdot\left(1+M_{X}(z)\right) & =M_{X}(z)\left[1+m_{X}(z)\right], \\
{\left[{ }^{c} T_{X}\left(m_{X}(z)\right)\right] \cdot\left(1+M_{X}(z)\right) } & =\frac{M_{X}(z)}{z} .
\end{aligned}
$$


With notation from Section 2 , for $\eta \in \mathcal{K}$ a non-zero vector and $f=\sum_{k=0}^{N} f_{k} X^{k}$ a polynomial with complex coefficients, we define $A_{\eta, f} \otimes$ via

$$
A_{\eta, f} \otimes \sum_{k=0}^{N} f_{k} \cdot A_{\eta, k} .
$$

\subsection{The ${ }^{c} R$-transform.}

Theorem 3.1. Let $\eta$ be a unit vector from $\mathcal{K}, b \in \pi(\mathcal{L}(\mathfrak{H}))$ and $f=\sum_{p=0}^{M} g_{p} \cdot z^{p}$ be a polynomial with complex coefficients. Consider $\alpha \in \mathcal{L}(\mathcal{E})$ given by

$$
\alpha=b+A_{\eta}^{*}+A_{\eta, f} \otimes .
$$

Then ${ }^{c} R_{\alpha}(z)=z f(z)$.

Proof. It suffices to show that $z f(z)$ satisfies equation (5), which is equivalent to the following recurrence:

(7) $\phi\left(\alpha^{n}\right)=\sum_{0 \leq p \leq n} \sum_{\substack{q_{1}, \ldots, q_{p} \geq 0 \\ n \geq 1+p+q_{1}+\cdots+q_{p}}} \phi\left(\alpha^{n-1-\left(p+q_{1}+\cdots+q_{p}\right)}\right) \cdot f_{p} \cdot \psi\left(\alpha^{q_{1}}\right) \cdots \psi\left(\alpha^{q_{p}}\right)$

for all $n>0$.

Let us denote $A=A_{\eta}^{*}$ and $B=A_{\eta, f} \otimes$. The triple $(b, A, B)$ satisfies the following relations:

$$
\begin{aligned}
& b \Omega=0, B(\mathcal{E})=\mathbb{C} \Omega, \\
& A b^{q} A=A \psi\left(b^{q}\right) A, B b^{q} A=B \psi\left(b^{q}\right) A \text { for all } q>0, \\
& \phi\left(B A^{n}\right)=f_{n}, \text { for all } n \geq 0
\end{aligned}
$$

(equations (8) and (9) are consequences of the relations from Remark 2.2 and (10) follows from Lemma 2.4).

Let $I=\{b, A, B\}$. Since $\alpha=\sum_{x \in I} x$, we have that

$$
\phi\left(\alpha^{n}\right)=\sum_{\left(x_{1}, \ldots, x_{n}\right) \in I^{n}} \phi\left(x_{n} x_{n-1} \cdots x_{1}\right) .
$$

To further simplify the writing, we introduce the following notation:

$$
I[n, j]=\left\{\left(x_{1}, \ldots, x_{n}\right) \in I^{n}, \min \left\{k: x_{k}=B\right\}=j\right\} .
$$

Since $b \Omega=0$ and $A(\mathcal{E}) \perp \Omega$, we have that $\phi\left(x_{n} \cdots x_{1}\right)=0$ unless $x_{n}=B$; hence $\left(x_{n}, \ldots, x_{1}\right) \in I[n, j]$ for some $j$. Also, for $\left(x_{n}, \ldots, x_{1}\right) \in I[n, j]$, since $B(\mathcal{E})=\mathbb{C} \Omega$, we have that $x_{j} \cdots x_{1} \Omega=\phi\left(x_{j} \cdots x_{1}\right)$, so $\phi\left(x_{n} \cdots x_{1}\right)=\phi\left(x_{n} \cdots x_{j+1}\right) \phi\left(x_{j} \cdots x_{1}\right)$. Therefore (11) becomes

$$
\begin{aligned}
\phi\left(\alpha^{n}\right) & =\sum_{j=1}^{n} \sum_{\left(x_{1}, \ldots, x_{n}\right) \in I[n, j]} \phi\left(x_{n} \cdots x_{1}\right) \\
& =\sum_{j=1}^{n} \sum_{\left(x_{1}, \ldots, x_{n}\right) \in I[n, j]} \phi\left(x_{n} \cdots x_{j+1}\right) \phi\left(x_{j} \cdots x_{1}\right) \\
& =\sum_{j=1}^{n} \sum_{\left(x_{1}, \ldots, x_{j}\right) \in I[j, j]} \phi\left(\alpha^{n-j}\right) \phi\left(x_{j} \cdots x_{1}\right) .
\end{aligned}
$$


Consider $\left(x_{1}, \ldots, x_{n}\right) \in I[n, n]$. If $n=1$, then $\phi\left(x_{n} \cdots x_{1}\right)=\phi(B)=f_{0}$. If $n>1$, then $x_{1} \Omega=0$ unless $x_{1}=A$. Let $1=k_{1}<\cdots<k_{p}<n$ be the set of all indices $k$ such that $x_{k}=A$. Letting $q_{j}=k_{j+1}-k_{j}-1, q_{p}=n-k_{p}-1$ and applying property (10), we obtain

$$
\begin{aligned}
\sum_{\left(x_{1}, \ldots, x_{n}\right) \in I[n, n]} \phi\left(x_{n} \cdots x_{1}\right) & =\sum_{p=1}^{n-1} \sum_{\substack{0 \leq q_{1}, \ldots, q_{p} \\
q_{1}+\cdots+q_{p}<n-p}} \phi\left(B \cdot \psi\left(b^{q_{p}}\right) \cdot A \cdots \psi\left(b^{q_{1}}\right) A\right) \\
& =\sum_{\substack{0 \leq q_{1}, \ldots, q_{p} \\
q_{1}+\cdots+q_{p}<n-p}} f_{p} \cdot \psi\left(b^{q_{p}}\right) \cdots \psi\left(b^{q_{1}}\right) .
\end{aligned}
$$

Finally, the equality $\psi\left(b^{q}\right)=\psi\left(\alpha^{q}\right)$ and equations (12), (13) imply (7), so the proof is complete.

\subsection{The ${ }^{c} T$-transform.}

Theorem 3.2. Let $\eta$ be a unit vector from $\mathcal{K}, d \in \pi(\mathcal{L}(\mathfrak{H}))$ and $f(z)=\sum_{k=0}^{M} f_{k} \cdot z^{k}$ be a polynomial with complex coefficients such that $\psi(b), f_{0} \neq 0$. Consider $\beta \in \mathcal{L}(\mathcal{E})$ given by

Then ${ }^{c} T_{\beta}(z)=f(z)$.

$$
\beta=d+d A_{\eta}^{*}+A_{\eta, f} \otimes
$$

Proof. The proof is similar to the one of Theorem 3.1. Again denote $A=A_{\eta}^{*}$, $B=A_{\eta, f} \otimes$ and consider the sets

$$
\begin{aligned}
& J=\{d, d A, B\}, \\
& J[n, l]=\left\{\left(x_{1}, \ldots, x_{n}\right) \in J^{n}, \min \left\{k: x_{k}=B\right\}=l\right\} .
\end{aligned}
$$

For $\left(x_{1}, \ldots, x_{n}\right) \in J^{n}$, we have that $\phi\left(x_{n} \cdots x_{1}\right)=0$ unless $x_{n}=B$; hence $\left(x_{1}, \ldots, x_{n}\right) \in J[n, l]$ for some $1 \leq l \leq n$. Also, note that equation (12) holds true if we replace $I$ with $J$; therefore

$$
\phi\left(\beta^{n}\right)=\sum_{l=1}^{n} \phi\left(\beta^{n-l}\right) \sum_{\left(x_{1}, \ldots, x_{l}\right) \in J[l, l]} \phi\left(x_{l} \cdots x_{1}\right) .
$$

Fix $n>0$ and let $\left(x_{1}, \ldots, x_{1}\right) \in J[n . n]$. If $n=1$, then $\phi\left(x_{n}, \ldots, x_{1}\right)=\phi(B)=f_{0}$. If $n=1$, then $\phi\left(x_{n} \cdots x_{1}\right)$ cancels unless $x_{1}=d A$. Let $1=k_{1}<k_{2}<\cdots<k_{p} \leq$ $n-1$ be the set of indices $k$ such that $x_{k}=d A$. Taking $q_{j}=k_{j+1}-k_{j}-1$ for $1<j<n-1$ and $q_{p}=n-k_{p}-1$, and applying property (10), we obtain

$$
\begin{aligned}
\sum_{\left(x_{1}, \ldots, x_{n}\right) \in J[n, n]} \phi\left(x_{n} \cdots x_{1}\right) & =\sum_{p=1}^{n-1} \sum_{\substack{0 \leq q_{1}, \ldots, q_{p} \\
q_{1}+\cdots+q_{p}<n}} \phi\left(B \cdot\left(b^{q_{p}-1}\right) \cdot d A \cdot b^{q_{p-1}-1} \cdots b^{q_{1}-1} \cdot d A\right) \\
& =\sum_{p=1}^{n-1} \sum_{\substack{0 \leq q_{1}, \ldots, q_{p} \\
q_{1}+\cdots+q_{p}<n}}\left(B \cdot \psi\left(d^{q_{p}}\right) \cdot A \cdot \psi\left(b^{q_{p-1}}\right) \cdots \psi\left(d^{q_{1}}\right) A\right) \\
& =\sum_{p=1}^{n-1} \sum_{\substack{0 \leq q_{1}, \ldots, q_{p} \\
q_{1}+\cdots+q_{p}<n}} f_{p} \cdot \psi\left(d^{q_{p}}\right) \cdots \psi\left(d^{q_{1}}\right) .
\end{aligned}
$$


The conclusion follows, since (15), (14) and the identity $\psi\left(\beta^{q}\right)=\psi\left(d^{q}\right)$ imply that the $f(z)$ satisfies (6).

\section{Addition AND multiplication of C-FREe RANDOM VARiables}

Theorem 4.1. Let $\eta_{1}, \eta_{2}$ be orthogonal unit vectors from $\mathcal{K}$; let $b_{1}, b_{2}, d_{1}, d_{2}$ be some elements from $\pi(\mathcal{L}(\mathfrak{H}))$; and let $f_{1}, f_{2}, F_{1}, F_{2}$ be polynomials with complex coefficients, such that $\psi\left(d_{i} \neq 0 \neq F_{i}(0)\right.$. Define $(i=1,2)$ :

$$
\begin{aligned}
& \alpha_{i}=b_{i}+A_{\eta_{i}}^{*}+A_{\eta_{i}, f_{i} \otimes}, \\
& \beta_{i}=d_{i}+d_{i} \cdot A_{\eta_{i}}^{*}+A_{\eta_{i}, F_{i}} \otimes
\end{aligned}
$$

Then ${ }^{c} R_{\alpha_{1}+\alpha_{2}}(z)={ }^{c} R_{\alpha_{1}}(z)+{ }^{c} R_{\alpha_{2}}(z)$ and ${ }^{c} T_{\beta_{1} \cdot \beta_{2}}(z)={ }^{c} T_{\beta_{1}}(z) \cdot{ }^{c} T_{\beta_{2}}(z)$.

Proof. Suppose that $F_{1}(z)=\sum_{k=0}^{M} h_{k} \cdot z^{k}$ and $F_{2}(z)=\sum_{k=0}^{M} l_{k} \cdot z^{k}$ (eventually $h_{M}$ or $l_{M}$ are zero).

To prove the first equality, we introduce the notation

$$
\begin{aligned}
\widetilde{b} & =b_{1}+b_{2}, \\
\widetilde{A} & =A_{\eta_{1}}^{*}+A_{\eta_{2}}^{*}, \\
\widetilde{B} & =A_{\eta_{1}, F_{1}^{\otimes}}+A_{\eta_{2}, F_{2}^{\otimes}} .
\end{aligned}
$$

Trivial verifications show that the triple $(\widetilde{b}, \widetilde{A}, \widetilde{B})$ verifies conditions (8)-(10). Therefore recurrence (17) holds true for $\alpha=\widetilde{b}+\widetilde{A}+\widetilde{B}=\alpha_{1}+\alpha_{2}$ and $\left\{g_{k}\right\}_{k=1}^{M}$ the coefficients of $f_{1}(z)+f_{2}(z)$, so ${ }^{c} R_{\alpha_{1}+\alpha_{2}}(z)=z\left[f_{1}(z)+f_{2}(z)\right]$, completing the proof.

For the second equality, we need to prove that $F_{1}(z) \cdot F_{2}(z)$ satisfies (6) for $\beta=\beta_{1} \cdot \beta_{2}$; that is, the recurrence formula

$$
\begin{aligned}
\phi\left(\beta^{n}\right) & =\sum_{p=0}^{n-1} \sum_{\substack{q_{1}, \ldots, q_{p}>0 \\
q_{1}+\cdots+q_{p} \leq n}} \phi\left(\beta^{n-1-\left(q_{1}+\cdots+q_{p}\right)}\right) \cdot\left[g_{p} \cdot \psi\left(\beta^{q_{p}}\right) \cdots \psi\left(\beta^{q_{1}}\right)\right] \\
& =\sum_{m=1}^{n} \phi\left(\beta^{n-m}\right) \cdot\left[\sum_{p=1}^{m-1} \sum_{\substack{q_{1}, \ldots, q_{p}>0 \\
q_{1}+\cdots+q_{p}<m}} g_{p} \cdot \psi\left(\beta^{q_{p}}\right) \cdots \psi\left(\beta^{q_{1}}\right)\right]
\end{aligned}
$$

is verified for $\beta=\beta_{1} \cdot \beta_{2}$ and $g_{m}$ the coefficient of $z^{m}$ in $F_{1}(z) \cdot F_{2}(z)$.

We introduce the notation

$$
\begin{aligned}
& b=d_{1} ; \quad d=d_{2}, \\
& A_{1}=A_{\eta_{1}}^{*} ; \quad A_{2}=A_{\eta_{2}}^{*}, \\
& B_{1}=A_{\eta_{1}, F_{1}^{\otimes}} ; \quad B_{2}=B_{\eta_{2}, F_{2}}^{\otimes} .
\end{aligned}
$$

Then $B_{i} A_{j}=0$ whenever $i \neq j$ and $\beta_{1}=b+b A_{1}+B_{1}, \beta_{2}=d+d A_{2}+B_{2}$. For $\beta=\beta_{1} \cdot \beta_{2}$, we have that

$$
\begin{aligned}
\beta & =\left(b+b A_{1}+B_{1}\right)\left(d+d A_{2}+B_{2}\right) \\
& =b d+b d A_{2}+b B_{2}+b A_{1} d+b A_{1} d A_{2}+b A_{1} B_{2}+B_{1} d+B_{1} d A_{2}+B_{1} B_{2} .
\end{aligned}
$$


Consider the sets

$$
\begin{aligned}
& \mathcal{J}=\left\{b d, b d A_{2}, b A_{1} d, b A_{1} d A_{2}, b A_{1} B_{2}, B_{1} d, B_{1} d A_{2}, B_{1} B_{2}\right\}, \\
& \mathcal{J}[n, m]=\left\{\left(x_{1}, \ldots, x_{n}\right) \in \mathcal{J}^{n}, \min _{k}\left\{x_{k} \in\left\{B_{1} d, B_{1} B_{2}\right\}\right\}=m\right\}, \\
& \overline{\mathcal{J}}=\mathcal{J} \cup\left\{b A_{1} d A_{2}\right\} .
\end{aligned}
$$

Note first that $b B_{2}=0$. Also, since Lemma 2.4 implies $B_{1} d A_{2}=B_{1} \psi(d) A_{2}=0$, we have that $\beta=\sum_{x \in \overline{\mathcal{J}}} x$; hence

$$
\phi\left(\beta^{n}\right)=\sum_{\left(x_{1}, \ldots, x_{n}\right) \in \overline{\mathcal{J}}} \phi\left(x_{n} \cdots x_{1}\right) .
$$

If some $x_{k}$ is $b A_{1} d A_{2}$, then $\phi\left(x_{n} \cdots x_{1}\right)$ cancels, since the vectors from $\mathcal{E}$ with the $\mathcal{T}(\mathcal{K})$ component containing mixed tensors in $\eta_{1}$ and $\eta_{2}$ are cancelled by any $B_{i}$ $(i=1,2)$ and are also orthogonal to $\Omega$. On the other hand, $\phi\left(x_{n} \cdots x_{1}\right)$ also cancels if $x_{n} \cdots x_{1} \perp \Omega$, that is, if $x_{n}$ does not start with some $B_{i}$. It follows that only terms having $x_{n} \in\left\{B_{1} d, B_{1} B_{2}\right\}$ contribute to the sum; that is, the sum can be taken only for $\left(x_{1}, \ldots, x_{n}\right) \in \mathcal{J}[n, m](1 \leq m \leq n)$.

Now consider $\left(x_{1}, \ldots, x_{n}\right) \in \mathcal{J}[n, m]$. Then $x_{m} \cdots x_{1} \Omega=\phi\left(x_{m} \cdots x_{1}\right) \Omega$; hence $\phi\left(x_{n} \cdots x_{1}\right)=\phi\left(x_{n} \cdots x_{m}\right) \phi\left(x_{m} \cdots x_{1}\right)$, and

$$
\begin{aligned}
\sum_{\left(x_{1}, \ldots, x_{n}\right) \in \mathcal{J}[n, m]} \phi\left(x_{n} \cdots x_{1}\right) & =\sum_{\left(x_{1}, \ldots, x_{n}\right) \in \mathcal{J}[n, m]} \phi\left(x_{n} \cdots x_{m+1}\right) \cdot \phi\left(x_{m} \cdots x_{1}\right) \\
& =\phi\left(\beta^{n-m}\right) \cdot\left[\sum_{\left(x_{1}, \ldots, x_{m}\right) \in \mathcal{J}[m, m]} \phi\left(x_{m} \cdots x_{1}\right)\right] .
\end{aligned}
$$

Therefore it suffices to prove that the second factors from the right hand sides of (17) coincide to the second factor of the $m$-th summand in (16); that is (we use that $\left.g_{m}=\sum_{p+k=m} l_{p} \cdot h_{k}\right)$,

$$
\begin{aligned}
\sum_{\left(x_{1}, \ldots, x_{n}\right) \in \mathcal{J}[n, n]} \phi\left(x_{n} \cdots x_{1}\right) & =\sum_{m=0}^{n-1} g_{m} \cdot \sum_{\substack{q_{1}, \ldots, q_{m}>0 \\
q_{1}+\cdots+q_{m}<n}} \psi\left(\beta^{q_{m}}\right) \cdots \psi\left(\beta^{q_{1}}\right) \\
& =\sum_{\substack{p, k \geq 0 \\
p+q<n}} E(p, k)
\end{aligned}
$$

for $E(p, k)=\left[l_{p} \psi\left(\beta^{q_{m}}\right) \cdots \psi\left(\beta^{q_{1}}\right)\right] \cdot\left[h_{k} \psi\left(\beta^{s_{k}}\right) \cdots \psi\left(\beta^{s_{1}}\right)\right.$, where the summation is done over all $p, q \geq 0$ such that $p+q<n$ and all $q_{1}, \ldots, q_{p}, s_{1}, \ldots s_{k}>0$ with $q_{1}+\cdots+q_{p}+s_{1}+\cdots+s_{k}=n-1$.

Consider $\left(x_{1}, \ldots, x_{n}\right) \in \mathcal{J}[n, n]$ such that $\phi\left(x_{n} \cdots x_{1}\right) \neq 0$; then $x_{1} \Omega \neq 0$, so $x_{1} \in\left\{B_{1} B_{2}, b A_{1} B_{2}, b d A_{2}\right\}$.

Case I: $x_{1}=B_{2} B_{2}$. This implies that $n=1$ (since $x_{1}$ already starts with a $B_{i}$ ) and $\phi\left(x_{1}\right)=\left\langle B_{1} B_{2} \Omega, \Omega\right\rangle=l_{0} h_{0}$, that is, (18) for $n=1$.

Case II: $x_{1}=b A_{1} B_{2}$. In this case $x_{1} \Omega=b A_{1} B_{2} \Omega=b A_{1} \Omega \cdot l_{0}$.

Let $j=\min \left\{k: x_{k}\right.$ contains $B_{1}$, i.e. $\left.x_{k} \in\left\{B_{1} B_{2}, B_{1} d\right\}\right\}$ (since $x_{n} \in\left\{B_{1} B_{2}, B_{1} d\right\}$, the set is not void). Also, $j \neq n$ will contradict the definition of $\mathcal{J}[n, n]$, so $j=n$.

Tensors containing $\eta_{1}$ are canceled by $B_{2}$ and, as seen earlier, summands with $A_{2}$ and $A_{1}$ not separated by $B_{1}$ do not contribute to the sum. Therefore it follows 
that $x_{2}, \ldots, x_{n-1}$ do not contain $A_{2}$ or $B_{2}$, so they can be only of the types $b d$ and $b A_{1} d$.

Let $1<k_{2}<\cdots<k_{p}<n$ be the indices of the factors of type $b A_{1} d$ and put $k_{1}=1$ and $k_{p+1}=n$. For $q_{i}=k_{i+1}-k_{i}$, applying Lemma 2.4, we have

$$
\begin{aligned}
x_{n} \cdots x_{2} b A_{1} \Omega & =B_{1} d(b d)^{q_{p}-1} b A_{1} d(b d)^{q_{p-1}-1} \cdots(b d)^{q_{1}-1} b A_{1} \Omega \\
& =B_{1} \psi\left((d b)^{q_{p}}\right) A_{1} \psi\left((d b)^{q_{p-1}}\right) \cdots \psi\left((d b)^{q_{1}}\right) A_{1} \Omega \\
& =\left(B_{1} A_{1}^{p} \Omega\right) \psi\left((d b)^{q_{p}}\right) \cdots \psi\left((d b)^{q_{1}}\right) \\
& =h_{p} \Omega \cdot \psi\left(\beta^{q_{p}}\right) \cdots \psi\left(\beta^{q_{1}}\right)
\end{aligned}
$$

since $B_{1} A_{1}^{p} \Omega=h_{p} \Omega$ and $\psi(d b)=\psi(b d)=\psi(\beta)$ due to the traciality of the vector states. Multiplying with $l_{0}$ and summing, we obtain

$$
\sum_{\substack{\left(x_{1}, \ldots, x_{n}\right) \\ x_{1}=d A_{1} B_{2}}} \phi\left(x_{n} \cdots x_{1}\right)=\sum_{p=1}^{n-1} E(p, 0) .
$$

Case III: $x_{1}=b d A_{2}$.

Let $x_{j}$ be the factor of the smallest index that contains $B_{2}$. Since $x_{j} \in \mathcal{J}$, we have that $x_{j}=y \cdot B_{2}$, with $y \in\left\{b A_{1}, B_{1}\right\}$.

None of the factors $x_{2}, \ldots, x_{j-1}$ contain $B_{1}$ (otherwise it will contradict the definition of $J[n, n])$. If some of them will contain $A_{1}$, then $y B_{2} x_{j-1} \cdots x_{2}=0$, since $B_{2}$ cancels all the tensors mixing $\eta_{1}$ and $\eta_{2}$. Hence $x_{2}, \ldots, x_{j-1} \in\left\{b d, b d A_{2}\right\}$. Again, let $1=j_{1}<\cdots<j_{k}<n$ be the indices of the factors of type $b d A_{2}$ and put $j_{k+1}=j$. For $s_{i}=k_{i+1}-k_{i}$, applying Lemma 2.4, we have

$$
\begin{aligned}
x_{j} \cdots x_{1} \Omega & =y \cdot B_{2}(b d)^{s_{k}} A_{2}(b d)^{s_{k-1}} A_{2} \cdots(b d)^{s_{1}} A_{2} \Omega \\
& =y \Omega \cdot l_{k} \psi(\beta)^{s_{k}} \cdots \psi\left(\beta^{s_{1}}\right)
\end{aligned}
$$

(we used that $B_{2} A_{2}^{k} \Omega=l_{k} \Omega$ and that $\psi\left(\beta^{s}\right)=\psi\left((b d)^{s}\right.$ ).

If $y=B_{1}$, then $y \Omega=h_{0}$; also, the minimality of $n$ implies $j=n$.

If $y=b A_{1}$, since $x_{n} \in\left\{B_{1} B_{2}, B_{1} d\right\}$, the minimality of $n$ implies that $x_{j+1}, \ldots$, $x_{n-1}$ do not contain $B_{1}$. If they do contain $A_{2}$ or $B_{2}$, then $\phi\left(x_{n} \cdots x_{1}\right)=0$ as seen earlier, so they must be of the types $b d$ or $b A_{1} d$. Also, if $x_{n}=B_{1} B_{2}$, then again $\phi\left(x_{n} \cdots x_{1}\right)=0$, so we can suppose $x_{n}=B_{1} d$. In this case, $x_{n} \cdots x_{j=1} y \Omega$ is in the setting of formula (19), so it is computed accordingly.

Summing, we obtain

$$
\sum_{\substack{\left(x_{1}, \ldots, x_{n}\right) \in \mathcal{J}[n, n] \\ x_{1}=b A_{2}}} \phi\left(x_{n} \cdots x_{1}\right)=\sum_{\substack{p, k-1>0 \\ p+k<n}} E(p, k),
$$

and the conclusion follows, since (22) and (20) imply (18).

Corollary 4.2. Let $\mathcal{A}$ be a unital algebra, $\Phi, \Psi: \mathcal{A} \longrightarrow \mathbb{C}$ be two linear maps with $\Phi(1)=\Psi(1)=1$ and let $X, Y$ be two c-free (with respect to the maps $\Phi, \Psi$ ) elements from $\mathcal{A}$.

(i) $R_{X+Y}=R_{X}+R_{X}$ and ${ }^{c} R_{X=Y}={ }^{c} R_{X}+{ }^{c} R_{Y}$ as formal power series.

(ii) If $\Psi(X), \Psi(Y)$ are non-zero, then $T_{X Y}=T_{X} \cdot T_{Y}$ and ${ }^{c} T_{X Y}={ }^{c} T_{X} \cdot{ }^{c} T_{Y}$ as formal power series. 
Proof. The equalities for $R$ - and $T$-transforms are basic properties in the Free Probability Theory (see [21], 13]). We need to prove (i) and (ii) for the ${ }^{c} R$ - and ${ }^{c} T$-transforms.

As in Corollary 2.7 we will consider two complex Hilbert spaces $\mathcal{H}$ and $\mathcal{K}$ of dimension at least two and $\mathcal{E}=\mathcal{T}(\mathcal{H}) \oplus[\mathcal{T}(\mathcal{H}) \otimes \mathcal{T}(\mathcal{K})]$, where

$$
\begin{aligned}
& \mathcal{T}(\mathcal{H})=\mathbb{C} \omega \oplus \mathcal{H} \oplus(\mathcal{H} \otimes \mathcal{H}) \oplus \ldots, \\
& \mathcal{T}(\mathcal{K})=\mathbb{C} \omega \oplus \mathcal{K} \oplus(\mathcal{K} \otimes \mathcal{K}) \oplus \ldots
\end{aligned}
$$

We fix $e_{1}, e_{2}$, respectively $\eta_{1}, \eta_{2}$, as two pairs of orthogonal unit vectors from $\mathcal{H}$, respectively $\mathcal{K}$. From Corollary 2.7, the algebras $\mathfrak{A}_{1}=\mathcal{D}\left(\eta_{1}\right) \vee \pi\left(\mathcal{A}\left(e_{1}\right)\right)$ and $\mathfrak{A}_{2}=$ $\mathcal{D}\left(\eta_{2}\right) \vee \pi\left(\mathcal{A}\left(e_{2}\right)\right)$ are c-free with respect to $\phi(\cdot)=\langle\cdot \omega \otimes \omega, \omega \otimes \omega\rangle$ and $\psi(\cdot)=\langle\cdot \omega, \omega\rangle$.

Also note that according to the relations defining the free, respectively c-free, independence (see subsection 2.1), the moments up to order $N$ of $X+Y$ and $X Y$ with respect to $\Phi$ and $\Psi$ are uniquely determined by the moments of order up to $N$ of $X$ and $Y$.

For (i), consider $f_{1}(z), F_{1}(z)$, respectively $f_{2}(z), F_{2}(z)$ to be the polynomials obtained by the truncation of order $N$ of $R_{X},{ }^{c} R_{X}$, respectively $R_{Y},{ }^{c} R_{Y}$ (i.e. if $c R_{X}(z)=\sum_{k=1}^{\infty} l_{k} \cdot z^{k}$, then $F_{1}(z)=\sum_{k=1}^{N} l_{k} \cdot z^{k}$ and the analogues).

With the notation from Section 2 , take $(i=1,2)$

$$
\alpha_{i}=\pi\left(a_{e_{i}}^{*}+f_{i}\left(a_{e_{i}}\right)\right)+A_{\eta_{i}}^{*}+A_{\eta_{i}, F_{i}^{\otimes}} .
$$

We have that $\alpha_{i} \in \mathfrak{A}_{i}$, so $\alpha_{1}, \alpha_{2}$ are c-free with respect to $\phi$ and $\psi$; hence, from Theorem 4.1 .

$$
{ }^{c} R_{\alpha_{1}+\alpha_{2}}(z)={ }^{c} R_{\alpha_{1}}(z)+{ }^{c} R_{\alpha_{2}}(z) .
$$

From Theorem 2.1 (2) and Theorem 3.1, we have that $R_{\alpha_{i}}(z)=f_{i}(z)$ and ${ }^{c} R_{\alpha_{i}}(z)=$ $F_{i}(z)$; therefore $R_{\alpha_{1}},{ }^{c} R_{\alpha_{1}}$, and $R_{X},{ }^{c} R_{X}$ coincide up to order $N$. Then equations (11) and (5) imply that the moments up to order $N$ of $X$ with respect to $\Phi$, respectively $\Psi$, coincide to the moments up to order $N$ of $\alpha_{1}$ with respect to $\phi$ and $\psi$. The same holds true for $Y$ and $\alpha_{2}$; therefore the moments up to order $N$ of $X+Y$ and $\alpha_{1}+\alpha_{2}$ do coincide. Henceforth, from equation (5), the first $N$ coefficients of ${ }^{c} R_{\alpha_{1}+\alpha_{2}}$ and ${ }^{c} R_{X+Y}$ do coincide. Since $N$ is arbitrary, equation (23) gives the conclusion.

The proof for (ii) is similar, taking $(i=1,2)$

$$
\beta_{i}=\pi\left(\left(\mathbf{I d}+a_{e_{i}}^{*}\right) f_{i}\left(a_{e_{i}}\right)\right)+\pi\left(\left(\mathbf{I d}+a_{e_{i}}^{*}\right) f_{i}\left(a_{e_{i}}\right)\right) A_{\eta_{i}}^{*}+A_{\eta_{i}, F_{i}^{\otimes}},
$$

where $f_{1}, F_{1}$, respectively $f_{2}, F_{2}$, are now the polynomials given by the truncation of order $N$ of $T_{X}$ and ${ }^{c} T_{X}$, respectively $T_{Y}$ and ${ }^{c} T_{Y}$.

\section{REFERENCES}

[1] Serban Teodor Belinschi, C-free convolution for measures with unbounded support, Von Neumann algebras in Sibiu, Theta Ser. Adv. Math., vol. 10, Theta, Bucharest, 2008, pp. 1-7. MR2512322(2010k:46068)

[2] Anis Ben Ghorbal and Michael Schürmann, Quantum stochastic calculus on Boolean Fock space, Infin. Dimens. Anal. Quantum Probab. Relat. Top. 7 (2004), no. 4, 631-650, DOI 10.1142/S0219025704001815. MR2105916 (2005j:81089)

[3] Florin Boca, Free products of completely positive maps and spectral sets, J. Funct. Anal. 97 (1991), no. 2, 251-263, DOI 10.1016/0022-1236(91)90001-L. MR.1111181 (92f:46064)

[4] Marek Bożejko, Uniformly bounded representations of free groups, J. Reine Angew. Math. 377 (1987), 170-186, DOI 10.1515/crll.1987.377.170. MR887407 (89a:22009) 
[5] Marek Bożejko, Positive definite functions on the free group and the noncommutative Riesz product (English, with Italian summary), Boll. Un. Mat. Ital. A (6) 5 (1986), no. 1, 13-21. MR.833375 (88a:43007)

[6] Marek Bożejko and Roland Speicher, $\psi$-independent and symmetrized white noises, Quantum probability \& related topics, QP-PQ, VI, World Sci. Publ., River Edge, NJ, 1991, pp. 219-236. MR.1149828

[7] Marek Bożejko, Michael Leinert, and Roland Speicher, Convolution and limit theorems for conditionally free random variables, Pacific J. Math. 175 (1996), no. 2, 357-388. MR.1432836 (98j:46069)

[8] Kenneth J. Dykema, Multilinear function series and transforms in free probability theory, Adv. Math. 208 (2007), no. 1, 351-407, DOI 10.1016/j.aim.2006.02.011. MR.2304321 (2008k:46193)

[9] Etienne F. Blanchard and Kenneth J. Dykema, Embeddings of reduced free products of operator algebras, Pacific J. Math. 199 (2001), no. 1, 1-19, DOI 10.2140/pjm.2001.199.1. MR,1847144 (2002f:46115)

[10] Uffe Haagerup, On Voiculescu's $R$ - and $S$-transforms for free non-commuting random variables, Free probability theory (Waterloo, ON, 1995), Fields Inst. Commun., vol. 12, Amer. Math. Soc., Providence, RI, 1997, pp. 127-148. MR1426838 (98c:46137)

[11] Anna Dorota Krystek, Infinite divisibility for the conditionally free convolution, Infin. Dimens. Anal. Quantum Probab. Relat. Top. 10 (2007), no. 4, 499-522, DOI 10.1142/S0219025707002919. MR2376439 (2009d:46118)

[12] Wojciech Młotkowski, Operator-valued version of conditionally free product, Studia Math. 153 (2002), no. 1, 13-30, DOI 10.4064/sm153-1-2. MR1948925 (2003j:46102)

[13] Alexandru Nica and Roland Speicher, Lectures on the combinatorics of free probability, London Mathematical Society Lecture Note Series, vol. 335, Cambridge University Press, Cambridge, 2006. MR2266879(2008k:46198)

[14] Mihai Popa, Multilinear function series in conditionally free probability with amalgamation, Commun. Stoch. Anal. 2 (2008), no. 2, 307-322. MR2446696 (2010i:46102)

[15] Mihai Popa, Realization of conditionally monotone independence and monotone products of completely positive maps, J. Operator Theory 68 (2012), no. 1, 257-274. MR2966045

[16] Mihai Popa, Non-crossing linked partitions and multiplication of free random variables (English summary), Operator theory live, Theta Ser. Adv. Math., vol. 12, pp. 135-143, Theta, Bucharest, 2010. MR2731869 (2012a:46128)

[17] Mihai Popa and Victor Vinnikov, Non-commutative functions and the non-commutative free Lévy-Hinčin formula, Adv. Math. 236 (2013), 131-157, DOI 10.1016/j.aim.2012.12.013. MR.3019719

[18] Mihai Popa and Jiun-Chau Wang, On multiplicative conditionally free convolution, Trans. Amer. Math. Soc. 363 (2011), no. 12, 6309-6335, DOI 10.1090/S0002-9947-2011-05242-6. MR2833556 (2012f:46134)

[19] Dan Voiculescu, Addition of certain noncommuting random variables, J. Funct. Anal. 66 (1986), no. 3, 323-346, DOI 10.1016/0022-1236(86)90062-5. MR839105 (87j:46122)

[20] Dan Voiculescu, Multiplication of certain noncommuting random variables, J. Operator Theory 18 (1987), no. 2, 223-235. MR915507 (89b:46076)

[21] D. V. Voiculescu, K. J. Dykema, and A. Nica, Free random variables, A noncommutative probability approach to free products with applications to random matrices, operator algebras and harmonic analysis on free groups, CRM Monograph Series, vol. 1, American Mathematical Society, Providence, RI, 1992. MR 1217253 (94c:46133)

[22] Jiun-Chau Wang, Limit theorems for additive conditionally free convolution, Canad. J. Math. 63 (2011), no. 1, 222-240, DOI 10.4153/CJM-2010-075-4. MR2779139(2012b:46145)

Center for Advanced Studies in Mathematics, Ben Gurion University of the Negev, P. O. Box 653, Be'er Sheva 84105, Israel; and Institute of Mathematics "Simion Stollow" of the Romanian Academy, P. O. Box 1-764, Bucharest, RO-70700, Romania

Current address: Department of Mathematics, The University of Texas at San Antonio, One UTSA Circle, San Antonio, Texas 78249-0664; and Institute of Mathematics "Simion Stoilow" of the Romanian Academy, P. O. Box 1-764, Bucharest, RO-70700, Romania

E-mail address: Mihai.Popa@utsa.edu 